\title{
Time-Dependent Critical Spin Fluctuations in Magnetic Salts
}

S W Lovesey

May 1995 
(C) Council for the Central Laboratory of the Research Councils 1995

Enquiries about copyright, reproduction and requests for additional copies of this report should be addressed to:

The Central Laboratory for the Research Councils

Library and Information Services

Rutherford Appleton Laboratory

Chilton

Didcot

Oxfordshire

OX11 OQX

Tel: 01235445384 Fax: 01235446403

E-mail library@rl.ac.uk

ISSN 1358-6254

Neither the Council nor the Laboratory accept any responsibility for loss or damage arising from the use of information contained in any of their reports or in any communication about their tests or investigations. 


\title{
Time-Dependent Critical Spin Fluctuations in Magnetic Salts
}

\author{
Stephen W. Lovesey, \\ ISIS Facility, Rutherford Appleton Laboratory, \\ Oxfordshire OX11 0QX, England.
}

\begin{abstract}
A short perspective of dynamic critical scattering is given, together with an orientation to modern theories of static and dynamic critical phenomena. The materials discussed are magnetic salts which are believed to spontaneously order through correlations induced by a Heisenberg exchange interaction between atomic spin moments.
\end{abstract}

Prepared for a special issue of the Canadian Journal of Physics in honour of Professor B. N. Brockhouse. 


\section{Introduction}

For a perfect paramagnet, in which there are no exchange interactions and no correlations between the magnetic ions, the magnetic neutron cross-section is strictly elastic, spatially isotropic, and independent of temperature. Inelasticity in the scattering from exchange coupled paramagnets at infinite temperature was studied first by Van Vleck [1], and he suggested the measurement of exchange-induced linewidths as a means of obtaining information about exchange forces in the solid state. Brockhouse [2], in a study of $\mathrm{Mn}_{2} \mathrm{O}_{3}$, reported the first such neutron scattering measurement. Van Vleck considered only single ion matrix elements in his calculation of the neutron interaction, but de Gennes [3] found a pronounced dependence of the energy distribution on the change in the neutron wavevector $\mathbf{k}$. This detailed, infinite temperature calculation was based on Van Hove's [4] representation of the neutron cross-section in terms of spin correlation functions. For large $\mathbf{k}$ de Gennes proposed a gaussian distribution changing to a truncated lorenzian distribution for small $\mathbf{k}$. The gross features of the temperature dependence of the cross-section are obtained on noting that all moments of the normalized energy spectrum are inversely proportional to the wavevector dependent isothermal susceptibility. At the critical temperature, $T_{c}$, this susceptibility diverges as $\mathbf{k}$ approaches a magnetic Bragg position. It follows that for such wavevectors there is a pronounced decrease in the inelasticity of the scattering near $T_{c}$, i.e. thermodynamic slowing down of long wavelength spin fluctuations.

The so-called conventional theory of scattering from exchange-coupled paramagnets outlined in the preceding paragraph has been reviewed by Marshall and Lowde [5]. It is not reliable at the critical temperature, where an infinite number of degrees of freedom are responsible for non-trivial features in the dynamics. Seminal work on this aspect of spin dynamics was reported by Wegner [6], Résibois and DeLeener [7] and Kawasaki [8]. It is now recognized, largely through the work of Hubbard [9], that these developments all lead to the same system of closed non-linear equations for the time-dependent and wave-vector-dependent spin-spin response function. Today, the set of equations are often referred to as the coupled-mode theory of the dynamical properties of spin systems.

Applied to critical spin dynamics, coupled-mode theory is in accord with two other powerful approaches. One, scaling theory, is a set of postulates that lead to predictions in the critical region from a knowledge of various properties in the hydrodynamical region. The renormalization-group method provides asymptotic properties of the spin-correlation function and explicit results for some critical 
exponents. Even though this method does not provide closed equations for the spinresponse function, it is particularly valuable since it alone is a systematic, perturbative approach to critical phenomena in spin systems, and other models which display a continuous phase transition. Results derived for spin systems using dynamic-scaling arguments and the renormalization-group method are gathered, together with copious references, by Privman et al. [10].

Although the pioneers of the coupled-mode approach concentrated attention on the critical properties of spin systems, there is a body of evidence to the effect that it provides an unrivalled account of paramagnetic fluctuations of short and long wavelengths in ferromagnetically coupled systems, e.g. EuO and EuS (Hubbard [9], Cuccoli et al. [11,12], Westhead et al. [13]). Perhaps the most recent example of the reliability of coupled-mode theory outside the critical region is in its application to a spin chain at infinite temperature (Lovesey and Balcar [14]). By and large, agreement between experimental and theoretical results for $\mathrm{RbMnF}_{3}$, an antiferromagnetically coupled system, is strikingly good. However, at $T_{c}$ and for $\mathbf{k}$ in the vicinity of the antiferromagnetic ordering wave vector there is an obvious disagreement: the experimental data for the energy spectrum shows a three-peaked structure, namely, an elastic (diffusive) component and two side peaks attributed to collective spin oscillations, albeit heavily damped oscillations, while theoretical results for the appropriate wave vectors show only two collective mode peaks [15].

\section{Preamble}

In the vicinity of a continuous phase transition (sometimes referred to as a second-order phase transition, and distinguished from a first-order phase transition by the absence of both hysteresis and a discontinuous change in the order parameter) fluctuations in the order parameter - magnetization of a ferromagnet - become extremely large. For an order-disorder transition, these fluctuations can be readily visualized as arising from the system locally making excursions between the two phases. So, for a ferromagnet very close to the critical temperature a small volume of the magnet fluctuates between an ordered, ferromagnetic state and a paramagnetic state. Such a process entails a very large number of spin excitations (normal modes or degrees of freedom) and this rises to an infinite number at $T_{c}$.

A continuous phase transition is experimentally characterized by the divergence at the critical point of the mean-square fluctuation in the order parameter, for this quantity, apart from some benign factors, is a response function. In the case of a ferromagnet the appropriate response function is the magnetic susceptibility, $\chi$, and if $\Delta M$ is the fluctuation in the magnetization $(\langle\Delta M\rangle=0$, where $<\ldots . .>$ denotes a thermal average), 


$$
\chi \quad \alpha<(\Delta M)^{2}>
$$

An external probe that couples to the order parameter displays a pronounced signature of the phase transition due to the divergence of $\chi$ at $T_{c}$. The latter is usually a power law; for a ferromagnet it is customary to use the notation,

$$
\chi \alpha\left(T-T_{\mathrm{c}}\right)^{-\gamma} ; T>T_{\mathfrak{c}}
$$

and the critical exponent $\gamma \sim 1.39$ for a simple, isotropic ferromagnet. Table I contains an abbreviated list of critical exponents, relations among the exponents (so-called scaling relations) and theoretically derived numerical values.

The foregoing comments refer to static properties of a system near a continuous phase transition. Equally dramatic effects are observed in the dynamic properties of the order parameter, scilicet, the associated relaxation time increases when the sample approaches the critical point. A simple, intuitive argument by which to interpret critical slowing down stems from considering the reversal of one spin. Near the critical point the susceptibility is very large, so the perturbation on the magnetic system caused by the spin reversal influences a large volume and hence a large number of spins (the perturbation in the magnet at a large distance from the reversed spin depends on distance $(r)$ like $\{\exp (-r / \xi) / r\}$, to a good approximation, where $\xi$ is the correlation length, Table $\mathrm{D}$ ). It naturally takes a very long time for thermal fluctuations to restore equilibrium by reversing each one of the large number of perturbed spins. A simple model of spin relaxation, which neglects couplings between the spin modes, predicts a relaxation time whose temperature dependence is provided by $\chi$, and this diverges at $T_{c}$.

At the time of writing, it is fair to say that static critical phenomena are well understood. From the viewpoint of theoretical work, there exists a complete formalism (renormalization group and conformal field theory) for the calculation of response functions and their associated critical exponents; a thorough account of this work is provided by Ma [16] and Zinn-Justin [17]. Hence, given a relatively simple model it is possible to confidently calculate static critical properties to a given accuracy. The same is not true of dynamic critical phenomena; at present, there is no complete computational formalism by which to obtain response functions observed in experiments using, say, neutron and photon beams. Indeed, it is a topic of research in which there is still much to be done; neutron beam (spin-echo, and scattering) techniques have much to offer. 
A topic which is not addressed here is the nature of the phase transition, and response functions, in spin glasses. It is fairly well established that, there exists a sharp transition in three-dimensional Ising spin glasses. Theoretical results for $3 \mathrm{~d}$ Heisenberg spin glasses are so far less conclusive (Fischer and Hertz [18]).

\section{Perspective}

Introductions to phase transitions and critical phenomena are given by Stanley [19], Als-Nielsen and Birgeneau [20] and Cardy [21]. An introduction and more advanced material, together with a survey of experimental results on magnetic systems, is provided by Collins [22]. Detailed accounts of theoretical work can be found in various articles in the series of books edited by Domb and Lebowitz, and the monographs by Ma [16], Patashinskii and Pokrovskii [23] and Zinn-Justin [17].

The nature of the strong critical scattering observed at a continuous phase transition depends on the spatial dimensionality of the magnetic system and the spin dimensionality. Indeed, the existence of a phase transition depends on these parameters; for example, a two-dimensional magnet with three-dimensional spins (planar Heisenberg) does not show long-range magnetic order at any finite temperature, in a contrast to a two-dimensional Ising-spin model which orders at a temperature whose magnitude is of the order of the exchange parameter. The behaviour of the susceptibility, and other static response functions, at the phase transition can be classified according to the space and the effective spin dimensionality, which recognizes magnetic anisotropies, and this feature of static critical phenomena is called universality. The latter is more complicated for dynamic critical phenomena. The term universality is justified by the fact that different physical systems with the same space and spin - or the variable(s) that corresponds to spin-dimensionality - display similar critical properties. A third feature that determines the universality class of a system is the nature of the interactions, and specifically their spatial range. For static critical phenomena it seems that just the three features of spin dimension, spatial dimension, and the range of the spin interactions completely determine the universality class.

Another important concept is that of scaling, which we choose to illustrate for quantities of direct relevance to the interpretation of scattering experiments. The fundamental information is that certain quantities are homogeneous functions of a few reduced variables. This information comes from the renormalization group method, although it can also be derived from a less rigorous line of reasoning. We restrict ourselves to consideration of the correlation length, Gibbs potential $(G)$ and the spin correlation function $\left(T \geq T_{c}\right)$, 


$$
C_{\mathrm{o}}(\mathbf{R})=\left\langle\Delta \mathbf{S}\left(\mathbf{R}+\mathbf{R}_{\mathrm{o}}\right) \cdot \Delta \mathbf{S}\left(\mathbf{R}_{\mathrm{o}}\right)\right\rangle
$$

where the spin deviation at site $\mathbf{R}$ is,

$$
\Delta \mathbf{S}(\mathbf{R})=\mathbf{S}_{\mathbf{R}}-<\mathbf{S}_{\mathbf{R}}>
$$

Concerning the definition of $C_{0}(\mathbf{R})$, it is independent of the arbitrary site vector $\mathbf{R}_{\mathrm{o}}$, and,

$$
\sum_{\mathbf{R}} C_{\mathbf{o}}(\mathbf{R})
$$

is proportional to the mean-square fluctuation in the magnetization considered in $\S 2$. For large values of $R=|\mathbf{R}|, C_{0}$ is essentially independent of the direction of $\mathbf{R}$.

The fundamental information is that $\xi, G$ and $C_{0}$ considered as functions of the reduced temperature $\tau=\left\{\left(T-T_{\mathrm{c}}\right) / T_{\mathrm{c}}\right\}$ and reduced magnetic field $h \propto\left(H / T_{c}\right)$ are homogeneous functions of the form,

$$
\begin{aligned}
& \xi(\tau, h)=\lambda \xi\left(\lambda^{y} \tau, \lambda^{x} h\right), \\
& G(\tau, h)=\lambda^{-d} G\left(\lambda^{y} \tau, \lambda^{x} h\right),
\end{aligned}
$$

and,

$$
C_{0}(R, \tau, h)=\lambda^{2(x-d)} C_{0}\left(\lambda^{-1} R, \lambda^{y} \tau, \lambda^{x} h\right)
$$

Here, $\lambda$ is a scaling variable, and the exponents $x$ and $y$ are related to those listed in Table I, as we will see. The physical concept expressed in these relations is that, at the critical point bulk quantities remain unchanged after scaling the variables. The scale variable, $\lambda$, is arbitrary, in other words there is a scale invariance, and it can be chosen at will. If $\lambda$ is a length the prefactors in the foregoing relations become self-evident; $\xi$ is a length, $G$ is a density, and $C_{0}$ is the product of spin densities derived from the second derivative of $G$ with respect to the field variable.

It is useful to follow the type of argument that yields scaling laws, i.e. relations between the critical exponents. First, let us establish $v y=1$. The definition of $v$ is $\xi \sim(1 / \tau)^{v}$ for $h=0$, cf. Table I. Turning to equation (1), we set $h=0$ and choose $\lambda^{y} \tau=1$, or $\lambda=(1 / \tau)^{1 / y}$; then, 


$$
\xi(\tau, 0)=(1 / \tau)^{1 / y} \xi(1,0)
$$

and the definition of $v$ leads immediately to the relation $v y=1$.

Having expressed the exponent $y$ in terms of one of the critical exponents listed in Table I, let us focus on the exponent $x$; we shall establish the relation,

$$
\gamma=v(2 x-d)
$$

which leads to the scaling relation cited in Table I between $\gamma, \eta$ and $v$. To obtain (5) we use the standard thermodynamic relation for the isothermal susceptibility as the second derivative of $G$ with respect to $h$ evaluated $h=0$, namely,

$$
\chi=-\left.\frac{\partial^{2}}{\partial h^{2}} G(\tau, h)\right|_{h=0}=-\left.(-1 / \tau)^{v(2 x-d)} \frac{\partial^{2}}{\partial h^{2}} G(-1, h)\right|_{h=0}
$$

The second equality follows from the choice,

$$
-\tau=(1 / \lambda)^{y}
$$

in which the sign of $\tau$ is appropriate for the ordered magnetic phase. If we assume that the critical exponent $\gamma$ is the same on both sides of the critical point, the relation (5) follows immediately from equation (6). (Exponents above and below $T_{c}$ are usually the same but the coefficients of proportionality, or critical amplitudes, are different, i.e. $G(1, h)$ is not expected to be the same as $G(-1, h)[10]$.)

In equation (3) we choose $\lambda=R$. For $h=0$, we have already established,

$$
R^{y} \tau \sim(R / \xi)^{y}
$$

so we can write,

$$
C_{\mathrm{o}}(R, \tau, 0)=R^{2(x-d)} C(R / \xi)
$$

where $C$ is a function of the dimensionless variable $(R / \xi)$ which contains the temperature dependence. The standard definition of the exponent $\eta$ is $\left(T=T_{c}\right)$,

$$
C_{\mathrm{o}}(R, 0,0) \sim R^{2-d-\eta},
$$

and it follows that (5) leads to the scaling relation, 


$$
\gamma=v(2-\eta)
$$

The relations (1) - (3) provide other scaling laws involving $\alpha$ and $\beta$ but we do not pause to obtain them.

Instead, we couch the foregoing discussion in terms of the wave vector dependent isothermal susceptibility, defined for a classical system as the spatial Fourier transform of $C_{0}(R, \tau, h)$. Denoting this important susceptibility by $\chi(k)$, equation (7) leads to the result $(h=0)$,

$$
\chi(k)=k^{\eta-2} \tilde{\mathrm{C}}(k \xi)
$$

where $\tilde{C}$ is proportional to the spatial Fourier transform of $C$. In particular, at $T_{c}$, where $k \xi \rightarrow \infty$,

$$
\chi(k) \sim k^{\eta-2} ; T=T_{c}
$$

In the limit $k \rightarrow 0, \chi(k)$ tends to the bulk susceptibility introduced in $\S 2$. From equation (10) it follows that, in this limit,

$$
\widetilde{\mathrm{C}}(k \xi) \sim(k \xi)^{2-\eta}
$$

and the identification of $\chi$ with $\chi(0)$ reproduces the scaling relation (9).

Let us now consider some dynamical properties of spin fluctuations in the vicinity of $T_{c}$. Results are less surely footed because there is not the solid base of information that (1) - (3) provide for static critical phenomena, i.e. the renormalization group method is less useful. There is an equally important method for dynamical properties called coupled-mode theory, which amounts to a certain recipe for obtaining self-consistent solutions to the full equation of motion for spin fluctuations.

The time-dependent generalization of the spatial Fourier transform of the spin correlation function $C_{0}(R)$ is denoted by $F(k, t)$. The equation of motion for $F(k, t)$, usually referred to as a generalized Langevin equation (see for example, Lovesey, [24]), shows that $F(k, t)$ is a homogeneous function that satisfies,

$$
F(k, t)=F\left(\lambda^{a} k, \lambda^{1 / 2} t\right)
$$

but the exponent $(a)$ is not determined. To find a value for this exponent it is necessary to invoke an approximation that closes the equation of motion. The 
aforementioned coupled-mode theory achieves closure, and produces an integrodifferential equation for $F(k, t)$ which is self-consistent, i.e. the dynamics is expressed solely in terms of $F(k, t)$. One can view a self-consistent equation as the result of a perturbation expansion for a subset of interactions taken to an infinite order. That such a type of approximation is required to generate physically sensible results in the critical region is a consequence of having, inevitably in an approximate manner, to account for the vast number of degrees of freedom that participate in critical fluctuations.

The standard form of the coupled-mode theory for a Heisenberg model of a ferromagnet gives the result $a=-1 / 5$, and the relation,

$$
F(k, t ; \xi)=F\left(\lambda^{a} k, \lambda^{1 / 2} t ; \lambda^{a} / \xi\right)
$$

In view of the relation (13) one is led to seek a self-consistent solution of the form,

$$
F(k, t ; \xi)=Q\left(k^{z} t \Omega(k \xi)\right)
$$

As in the case of static critical phenomena, useful information can be extracted from (14) without knowledge of the single-variable functions $Q$ and $\Omega$. Such knowledge is required, however, when coupled-mode theory is confronted with the spectrum of spin fluctuations observed by neutron spectroscopy (Cuccoli et al. [11], [12]).

Equations (13) and (14) are mutually consistent when,

$$
a z+\frac{1}{2}=0
$$

Since $a=-1 / 5$ the dynamic critical exponent $z=5 / 2$. At the critical point $(k \xi) \rightarrow \infty$, and $\Omega(k \xi)$ approaches a constant. In consequence, the relaxation rate for ferromagnetic spin fluctuations $\Gamma(k) \alpha k^{5 / 2}$ at $T=T_{c}$. The data obtained for EuO at $T_{c}$, displayed in fig. (1), is consistent with the value $z=5 / 2$ over a wide range of wave vectors. By carrying through the self-consistent solution of the coupled-mode theory one finds $(h=0)$,

$$
\Gamma(k)=k^{5 / 2}(2 / 3 \pi)\left(T_{c} c v_{\mathrm{o}}\right)^{1 / 2} ; T=T_{c},
$$

where $c$ and $v_{0}$ are the exchange stiffness and unit cell volume, respectively (the spinwave stiffness $D=2 c S$ ). 
We conclude this section by considering the influence of dipolar forces between the atomic magnetic moments. Such forces are present in all magnetic materials, of course, and produce the effect of demagnetization. A measure of the strength of dipolar forces is the wave vector $q_{d}$ defined by,

$$
\left(2 \pi / \nu_{\mathrm{o}}\right)\left(g \mu_{\mathrm{B}}\right)^{2}=c q_{d}^{2}
$$

in which $g$ is the gyromagnetic factor. For the moment, it is useful to note the values of $q_{d}$ for EuO and EuS, namely, $0.15 \AA^{-1}(\mathrm{EuO})$ and $0.26 \AA^{-1}$ (EuS), from which it is seen that dipolar forces are larger in EuS than EuO. Experiments by Böni et al. [25, 26] on EuS are largely consistent with theoretical results derived by Frey and Schwabl [27] from coupled-mode theory applied to a Heisenberg Hamiltonian including dipolar forces.

The inclusion of dipolar forces produces spatial anisotropy. For $T \geq T_{c}$ it is logical to speak of fluctuations parallel (longitudinal) and perpendicular (transverse) to the wave vector $\mathbf{k}$ associated with the spin fluctuations under observation. One effect of the dipolar forces between atomic moments is to prevent the longitudinal fluctuations from diverging in the critical region, $k \rightarrow 0$ and $T \rightarrow T_{c}$, so the phase transition is dominated by transverse fluctuations. The relaxation rates are found to be,

$$
\Gamma_{\alpha}\left(k, q_{d}\right) \alpha k^{5 / 2} \gamma_{\alpha}(x, y) ; \alpha=L, T
$$

where the dimensionless variables $x, y$ are,

$$
x=(1 / k \xi), \text { and } y=\left(q_{d} / k\right)
$$

Table II contains the asymptotic behaviour of the scaling functions $\gamma_{\alpha}(x, y)$, in which the notation is: $D$, dipolar; $I$, isotropic; $C$, critical; $H$, hydrodynamic. The four limiting regions are then: DC, $x<<1, y>>1$; IC, $x<<1, y<<1$; DH, $x>>1, y>>x$; IH, $x>>1, y<<x$.

The dynamic critical exponent of the longitudinal relaxation rate is found to cross-over from $z=5 / 2$ in the isotropic critical region, considered in previous paragraphs, to $z=0$ in the dipolar critical region. The situation for transverse fluctuations is a cross-over from $z=5 / 2$ to $z=2$. A numerical solution of the coupledmode equations shows that for transverse fluctuations the dynamic cross-over is shifted with respect to the static one, $k \sim q_{d}$, to a wave vector $\sim q_{d} / 10$. Longitudinal fluctuations are not anomalous and the dynamic and static cross-overs coincide. The 
dynamic cross-over in the transverse fluctuations has not been clearly demonstrated experimentally.

\section{Neutron Scattering}

The neutron scattering cross-section is proportional to $(\alpha, \beta$ label Cartesian components),

$$
\sum_{\alpha, \beta}\left(\delta_{\alpha \beta}-\kappa_{\alpha} \kappa_{\beta} / \kappa^{2}\right) S^{\alpha \beta}(\kappa, \omega),
$$

where the so-called van Hove response function for a sample with $N$ spins on a lattice is,

$$
S^{\alpha \beta}(\kappa, \omega)=\frac{1}{2 \pi N} \int_{-\infty}^{\infty} \mathrm{dt} \exp (-\mathrm{i} \omega t) \sum_{\mathbf{R}, \mathbf{R}^{\prime}} \exp \left\{\mathrm{i \kappa} .\left(\mathbf{R}-\mathbf{R}^{\prime}\right)\right\}\left\langle\Delta S^{\alpha}(\mathbf{R}, 0) \Delta S^{\beta}\left(\mathbf{R}^{\prime}, t\right)\right\rangle
$$

Recall that the spin fluctuation at the site labelled $\mathbf{R}$ satisfies $\left\langle\Delta S^{\alpha}(\mathbf{R})\right\rangle=0$, while $\Delta S(\mathbf{R}, t)$ is a Heisenberg operator at the time $t$. Inspection of (16) reveals that $S^{\alpha \beta}(\kappa, \omega)$ is the spatial and temporal Fourier transform of spin autocorrelation functions.

Let us now make contact between the measured response function and quantities introduced in previous sections. First, the integral of $S^{\alpha \beta}(\mathbf{k}, \omega)$ with respect to $\omega$ is proportional to the isothermal susceptibility, cf. equation (10) that applies to an isotropic system for which the spin autocorrelation function vanishes for $\alpha \neq \beta$, while for $\alpha=\beta$ it is independent of the value of the Cartesian label. The experimental realization of integrating over $\omega$, to measure the total scattering, is discussed by Collins [22] and Als-Nielsen [28].

It is now almost standard practice to decompose $S^{\alpha \beta}(\mathbf{k}, \omega)$ in the following manner,

$$
S(\mathbf{k}, \omega)=T \chi(\mathbf{k}) F(\mathbf{k}, \omega)
$$

where for simplicity we omit the Cartesian labels on $S(\mathbf{k}, \omega), \chi(\mathbf{k})$ and $F(\mathbf{k}, \omega)$, and the latter is the time Fourier transform of $F(\mathbf{k}, t)$ introduced in $\$ 3$.

Near $T_{c}$, and neglecting the critical exponent $\eta$, the susceptibility of an isotropic spin system is well represented by the form adopted by Ornstein and Zernike in which, 


$$
\chi(k) \sim\left\{(k \xi)^{2}+1\right\}^{-1}
$$

The expression appropriate in the presence of dipolar forces is given by Frey and Schwabl [27].

A simple derivation of the coupled-mode equation for $F(\mathbf{k}, t)$, together with an examination of its predictions, is given by Lovesey [24]. Complete solutions to the equation, obtained by numerical integration, are applied by Cuccoli et al. $[11,12]$ to the interpretation of neutron scattering data obtained for $T \geq T_{c}$ and a wide range of wave vectors. The confrontation of theory and data provides good evidence for the success of the coupled-mode approximation for the description of paramagnetic and critical spin fluctuations in ferromagnetically coupled systems. A similar finding is reached by Frey and Schwabl [27] in their discussion of effects due to dipolar forces; see, also, [29].

Experimental results on the relaxation rate $\Gamma(k)$ are usually obtained by fitting a model $S(k, \omega)$ to the data. This is most often accomplished by use of the susceptibility (18) and,

$$
\pi \Gamma F(k, \omega)=\left(\omega^{2}+\Gamma^{2}\right)^{-1},
$$

where $\Gamma=\Gamma(k)$ is discussed in $\S 3$. Ideally, one would like to use a more firmly based approximation for $F(k, \omega)$. Certainly, it is required to test results for $\Gamma(k)$ for their dependence on the assumed shape of $F(k, \omega)$. At present, the computer time involved in solving the coupled-mode equation for $F(k, t)$ is too large for a direct fitting scheme to be a practical exercise, and so some form of parameterization, such as (19), seems unavoidable.

One can speculate that the apparent disagreement between experimental data on an antiferromagnetically coupled system, $\mathrm{RbMnF}_{3}$, and results from coupled-mode theory applied to an appropriate model [15] is due to this kind of fitting scheme. Data at $T_{c}$ seem to support the existence of a central, diffusive component in $F(k, \omega)$ observed at the antiferromagnetic ordering wave vector. In contrast, theoretical results for $F(k, \omega)$ displayed in fig. (2) have a dip at the elastic $(\omega=0)$ position.

\section{Conclusions}

The investigation of time-dependent critical and paramagnetic spin fluctuations by neutron beam techniques, which was started in 1955 by Brockhouse, 
has been very influential in the development of sophisticated modern theories of cooperative phenomena. Neutron beam techniques, and a few other experimental probes, have the great advantage of providing good quality data on quantities that are central to theoretical developments. It appears that, static critical phenomena are largely understood, and theoretical methods are surely footed. The same situation does not prevail with regard to dynamic critical phenomena. Not surprisingly, neutron beam data is needed to shed light on the known outstanding issues in this field of basic research.

\section{Acknowledgement}

Dr. R. Vaia gave valuable critical comments on an early draft of the paper. 


\section{Table I}

Magnetization $\sim\left(T_{c}-T\right)^{\beta}$

Susceptibility, $\chi \sim\left(T-T_{c}\right)^{-\gamma}$

Correlation length, $\xi \sim\left(T-T_{c}\right)^{-v}$

Specific heat $\sim\left(T-T_{c}\right)^{-\alpha}$

Fisher exponent, $\eta: \gamma=v(2-\eta)$

$$
\begin{aligned}
& \alpha=2-v d \quad) \quad d=\text { spatial dimension } \\
& 2 \beta=v(d-2+\eta))
\end{aligned}
$$

Isotropic ferromagnet, $d=3$ : critical exponents derived from a renormalization group calculation (from Zinn-Justin [17] table 25.6)

$$
\begin{aligned}
& \beta=0.368 \pm 0.004 \\
& \gamma=1.390 \pm 0.010 \\
& v=0.710 \pm 0.007 \\
& \eta=0.040 \pm 0.003
\end{aligned}
$$




\section{Table II}

\begin{tabular}{lc|l} 
& $\gamma_{\mathrm{T}}$ & $\gamma_{\mathrm{L}}$ \\
\hline $\mathrm{DC}$ & $y^{1 / 2}$ & $y^{5 / 2}$ \\
$\mathrm{IC}$ & 1 & 1 \\
$\mathrm{DH}$ & $x^{2} y^{1 / 2}$ & $y^{5 / 2}$ \\
$\mathrm{IH}$ & $x^{1 / 2}$ & $x^{1 / 2}$
\end{tabular}

Asymplotic behaviour of the scaling functions for the relaxation rates in a paramagnet in which the dipolar energy is included: After Frey and Schwabl [27]; see, also, [29]. 


\section{References}

1. J. H. Van Vleck, Phys. Rev. 53, 924-30, (1939).

2. B. N. Brockhouse, Phys. Rev. 99, 601, (1955).

3. P. G. De Gennes, J. Phys. Chem. Solids 4, 223-6, (1958).

4. L. Van Hove, Phys. Rev. 95, 249-62, (1954).

5. W. Marshall, and R. D. Lowde, Rep. Prog. Phys. 31, 705, (1968).

6. F. Wegner, Z. Phys. 218, 260, (1969).

7. P. Résibois, and M. De Leener, Phys. Rev. 178, 806, 819, (1969).

8. K. Kawasaki, Ann. Phys. (N.Y.) 61, 1, (1970).

9. J. Hubbard, Phys. C: Solid State Phys. 4, 53, (1971).

10. V.Privman, P. C. Hohenberg, and A. Aharony, Phase Transitions and Critical Phenomena vol 14, ed C. Domb and J. L. Lebowitz, London: Academic (1990).

11. A. Cuccoli, V. Tognetti, and S. W. Lovesey, Phys. Rev. B39, 2619, (1989).

12. A. Cuccoli, V. Tognetti, and S. W. Lovesey, J. Phys.: Condens. Matter 2, 3339, (1990).

13. D. R. Westhead, A. Cuccoli, S. W. Lovesey, and V. Tognetti, J. Phys.: Condens. Matter 3, 5235, (1991).

14. S. W. Lovesey, and E. Balcar, J. Phys.: Condens. Matter 6, 1253, (1994).

15. A. Cuccoli, S. W. Lovesey, and V. Tognetti, J. Phys.: Condens. Matter 6, 7553 (1994)

16. S. K. Ma, Modern Theory of Critical Phenomena, Vol. 46, Frontiers in Physics. Benjamin/Cummings Publishing, California (1976).

17. J. Zinn-Justin, Quantum Field Theory and Critical Phenomena. Oxford University Press (1990)

18. K. H. Fischer, and J. A. Hertz, Spin Glasses. Cambridge University Press (1991)

19. Stanley, H. E. (1971) Phase Transitions and Critical Phenomena. Oxford University Press.

20. J. Als-Nielsen, and R.J. Birgeneau, Am. J. Phys. 45, 554 (1977).

21. J. Cardy, Physics World, pg. 29, June (1993). 
22. M. F. Collins, Magnetic Critical Scattering. Oxford University Press (1989).

23. A. Z. Patashinskii, and V. L. Pokrovskii, Fluctuation Theory of Phase Transitions. Pergamon Press, Oxford (1979).

24. S.W. Lovesey, Condensed Matter Physics: Dynamic Correlations, 2nd. edition, Frontiers in Physics Vol. 61. Benjamin/Cummings Publishing, California (1986).

25. P. Böni, M. E. Chen, and G. Shirane, Phys. Rev. B35, 8449 (1987).

26. P. Boni, D. Görlitz, J. Kötzler, and J. L. Martínez, Phys. Rev. B43, 8755 (1991).

27. E. Frey, and F. Schwabl, Z. Phys. B71, 355 (1988).

28. J. Als-Nielsen, Phase Transitions and Critical Phenomena vol 5a, ed C. Domb and M. S. Green, London Academic (1974).

29. S. V. Maleev, Sov. Sci. Rev. A. Phys., 8, 323 (1987).

30. P. Böni and G. Shirane, Phys. Rev. B33, 3012 (1986). 


\section{Figure Captions}

1. Logarithmic plot of the relaxation rate of the critical fluctuations $\left(T=T_{c}\right)$ as a function of wavevector $\mathbf{q}$ in EuO. The data follow a variation with $q^{z}, z=5 / 2$, over four decades of energy [30].

2. $F(\mathbf{k}, \omega)$ is displayed for $T=T_{c}$; results are derived from coupled-mode theory applied to a model of $\mathrm{RbMnF}_{3}$ [15]. The wavevector $\mathbf{k}$ is close to the Brillouin zone centre in panel (a), and close to the antiferromagnetic-ordering wavevector, $w=(1,1,1)$ in panel (b). The wavevectors in units of $\left(\pi / 12 a_{0}\right)$ are: $1,(0,0,1) ; 2,(0,1,1) ; 3,(1,1,1) ; 4,(11,12,12)$ $; 5,(11,11,12)$ and $6,(11,11,11)$. 


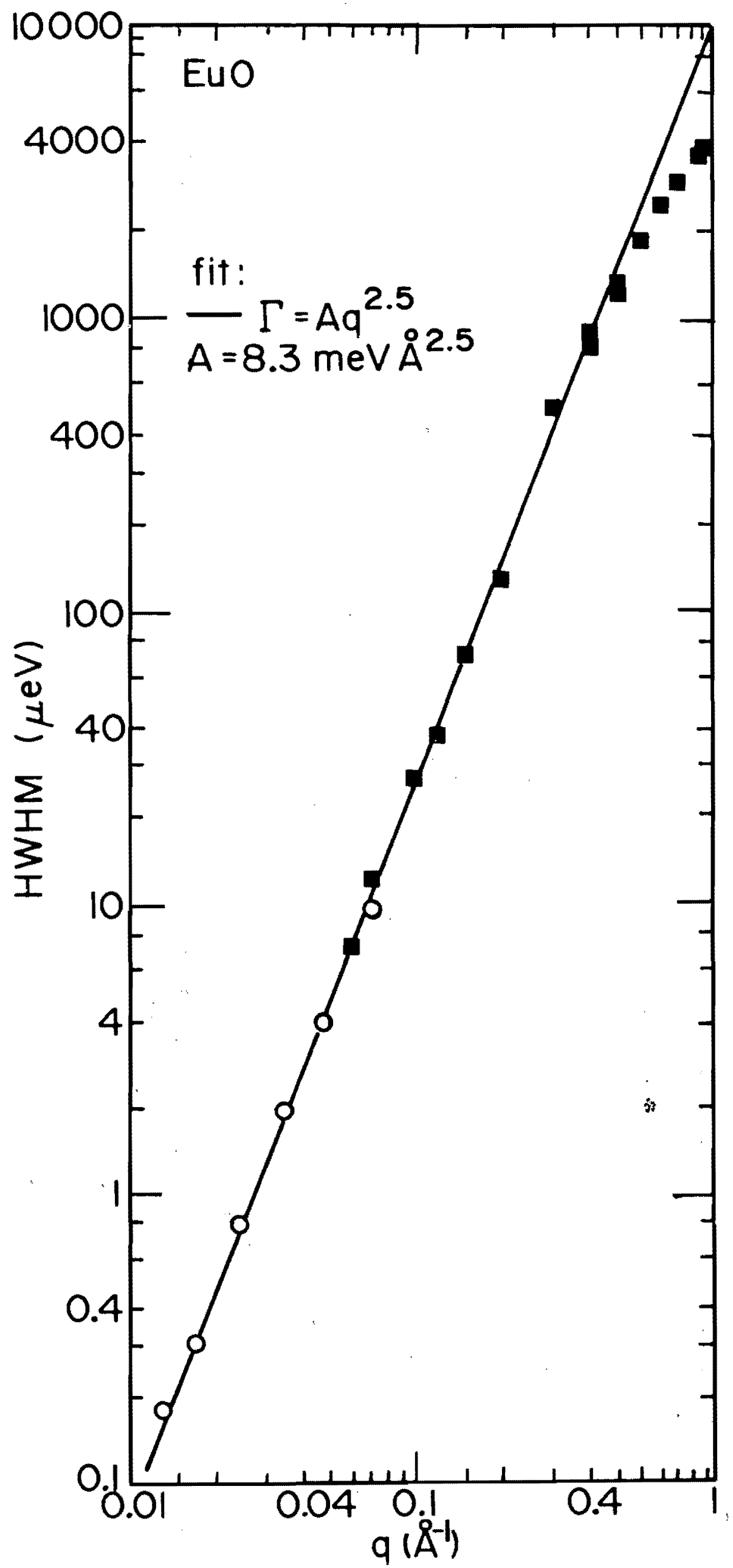




$$
\mathbf{T}=\mathbf{T}_{\mathbf{c}}
$$

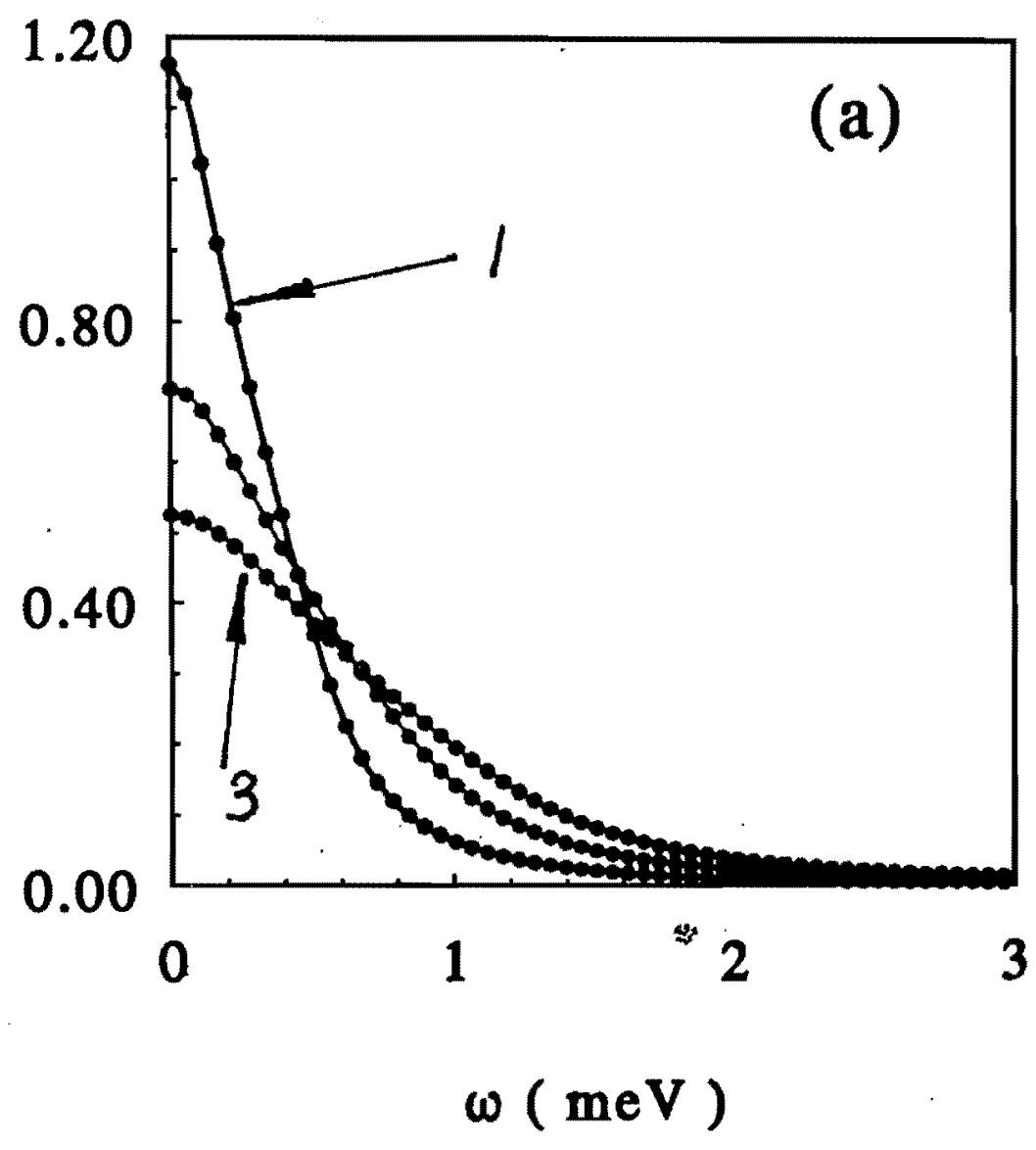

$\mathbf{T}=\mathbf{T}_{\mathrm{c}}$

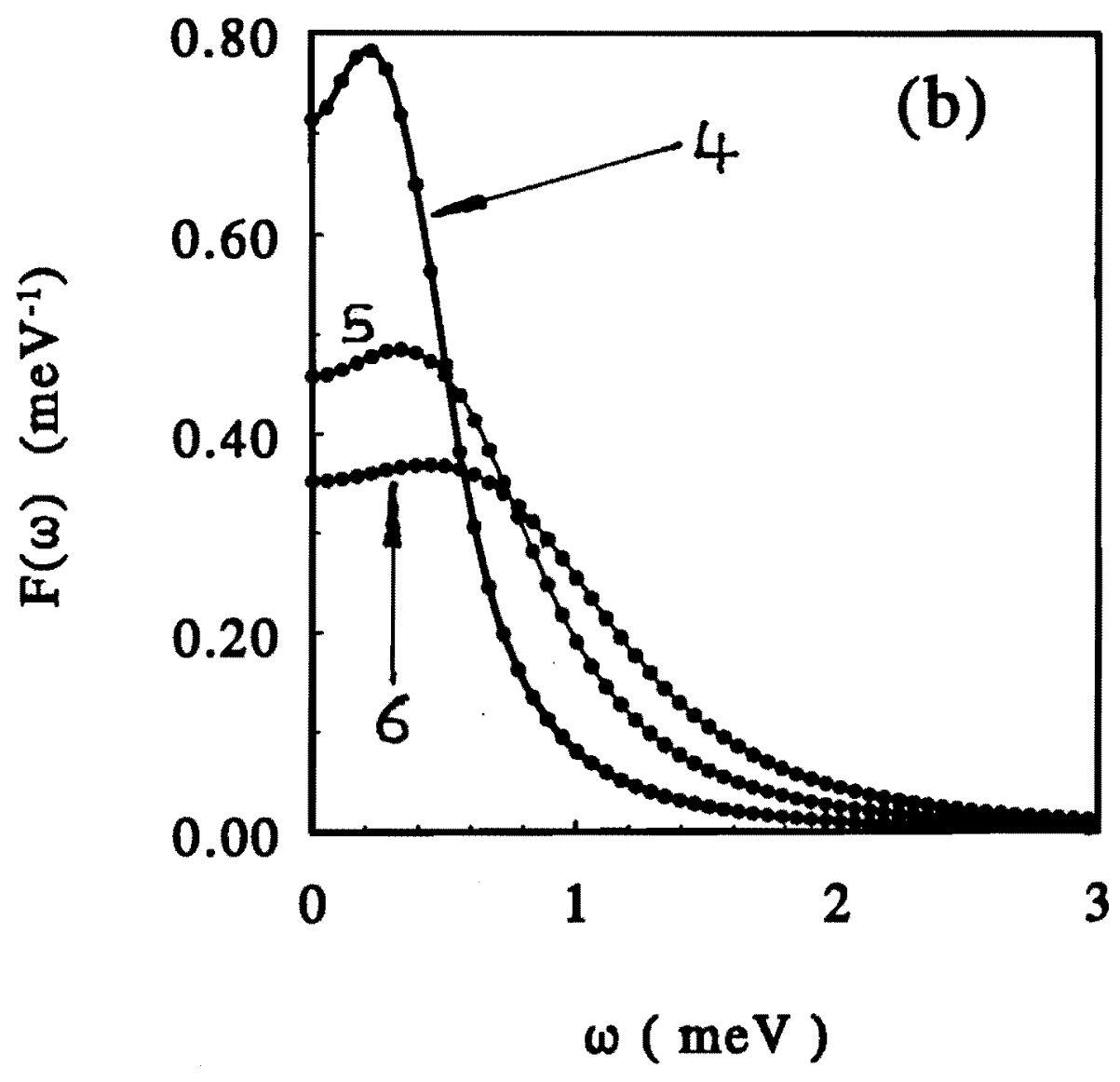

